\title{
CORRIGENDUM
}

to the paper

\section{ON SOME CLASSES OF WEIGHTED COMPOSITION OPERATORS}

\author{
by JAMES T. CAMPBELL and JAMES E. JAMISON
}

(Received 7 March, 1990)

0. Abstract. We provide corrected versions of Theorems 1 and 2 and Corollaries 3 (a) and 4 of the paper mentioned in the title.

I. Basic results. In our paper [2], Theorems 1 and 2 and Corollaries 3(a) and 4 contain errors. Assuming the notation of that paper the following are correct versions of Theorems 1 and 2 , respectively.

THEOREM I. $W_{T}$ is normal if and only if

and

(i) $w E(w) h \circ T=h E\left(w^{2}\right) \circ T^{-1}$ a.e.

(ii) $T^{-1} \Sigma \cap \operatorname{supp} w=\Sigma \cap \operatorname{supp} w$.

THEOREM II. $W_{T}$ is quasinormal if and only if $h \circ T E\left(w^{2}\right)=h E\left(w^{2}\right) \circ T^{-1}$ a.e. on the support of $w$.

RemarKs. 1. For the proofs of these theorems, as well as other examples, see [3].

2. The original attempt to prove Theorem 2 in [2] yields Theorem II provided one observes a factor of $w$ in each term of $V M$ and $M V$, where $M=\left|W_{T} * W_{T}\right|^{1 / 2}$ and $V$ is the partial isometry which gives the unique, canonical polar form $V M=W_{T}$.

3. The fallacy in the proof of Theorem 1 in [2] was the claim that for a normal $W_{T}$, the set $A=$ support of $w$ (written supp $w$ ) satisfied $T^{-1} A=A$. This was never proved and may in fact be false; see Example 1 of [3], or Example 1 below.

4. All the other results in [2] except Corollaries 3(a) and 4, which we deal with below, are correct.

Here is a corrected statement and proof of Corollary 3(a) from [2]. For ease of proof we assume $w \geq 0$ a.e. . The general complex case is clear and easily obtained.

Corollary 3(a). Suppose $T$ is a non-invertible, conservative and ergodic measurepreserving transformation. Then $W_{T}$ is not normal for any (non-zero) choice of $w$.

Proof. Given such a $T$, suppose $W_{T}$ is normal. It follows (see [3]) that $A$, the support of $w$, satisfies $A \subseteq T^{-1} A$. Since $T$ is conservative, it must be the case that $A=T^{-1} A$. Since $T$ is ergodic we have either $\mu(A)=0$ or $\mu(X \backslash A)=0$. In the first case $w=0$. In the second case, by (ii) of the above Theorem II, $T$ must be invertible.

REMARK. In the case of finite measure, every measure-preserving transformation is conservative, and this Corollary was know to Bastian (although his proof rests on different principles; see [1]). In any case, it presents an interesting dichotomy for

Glasgow Math. J. 32 (1990) 261-263. 
measure-preserving transformations. If a measure-preserving transformation $T$ is invertible, the composition operator it induces is unitary; however if $T$ is not invertible the induced composition operator is not even normal. Corollary 3(a) says that for such a $T$, being conservative and ergodic implies that the composition operator cannot even be weighted to become normal. We do not know what is possible for non-conservative $T$.

We turn now to Corollary 4 of [2], which attempts to characterize the Hermitian weighted composition operators. We have the following example.

Example 1. Let $X=\{0,1,2\}, \Sigma=2^{X}, \mu(x)=1$ for each $x \in X$. Define $T: X \rightarrow X$ by $T(0)=1, T(1)=2$, and $T(2)=1$. Set $w=\chi_{A}$, where $A=\{1,2\}$. Then direct calculations show that for all $f \in L^{2}(X), W_{T} f(0)=0, W_{T} f(1)=f(2)$, and $W_{T} f(2)=f(1)$. Also $W_{T}^{*} f(0)=h(0) E(w f) \circ T^{-1}(0)=0$, and similarly $W_{T}^{*} f(1)=f(2), W_{T}^{*} f(2)=f(1)$, so that $W_{T}$ is hermitian. However, $T$ is not of period 2 (in fact $T$ is not invertible) and $h \bar{w} \circ T \neq w$ (in fact $w$ is not even $T^{-1} \Sigma$ measurable), contradicting Corollary 4 of [2].

The aspect of the proof of Corollary 4 in [2] which fails is again the fact that the support of the weight function may not be invariant under $T$. We resolve this in the following manner.

Given $W_{T}$, set $A=$ support of $w$ and define $T_{A}$ as the restriction of $T$ to $A$. Thus if $B \subseteq A, T_{A}^{-1} B=T^{-1} B \cap A$.

Corollary 4. $W_{T}$ is Hermitian if and only if

(i) $T_{A}$ is periodic of period 2

and

(ii) $w=h E(\bar{w}) \circ T^{-1}$.

Proof. Suppose $W_{T}$ is Hermitian; then $W_{T}$ is normal. Thus (see [3]) we know that $T$ maps $A$ into $A$ (so that $\left.A \subseteq T^{-1} A\right), L^{2}(A)$ is reducing for $W_{T}$, $\operatorname{ker} W_{T}=L^{2}(X \backslash A)$, and $A=\operatorname{supp} h E\left(|w|^{2}\right) \circ T^{-1}$.

Setting $W_{T}=W_{T}^{*}$ yields

(1) $w f \circ T=h E(\bar{w} f) \circ T^{-1}$ for all $f \in L^{2}(X, \Sigma, \mu)$.

Choose an increasing sequence of measurable sets $\left\{C_{n}\right\}$, each of finite measure, whose union is all of $X$. Setting $f=\chi_{C_{n}}$ in (1) and letting $n \rightarrow \infty$ we obtain (ii). Since $W_{T}^{2}=W_{T}^{*} W_{T}$ we obtain

(2) $w w \circ T f \circ T^{2}=h E\left(|w|^{2}\right) \circ T^{-1} f$, for all $f \in L^{2}(A)$.

Now choose an increasing sequence of measurable subsets $\left\{C_{n}\right\}$ of $A$, each of finite measure, whose union is all of $A$. Setting $f=\chi_{C_{n}}$ in (2) and letting $n \rightarrow \infty$ we obtain

(3) $w w \circ T=h E\left(|w|^{2}\right) \circ T^{-1}$.

Dividing both sides of (2) by $w w \circ T$ (since both sides are supported in $A$ we leave everything 0 off of $A$ ) yields

(4) $\chi_{A} f \circ T^{2}=f$ for all $f \in L^{2}(A)$.

In particular, for each measurable subset $C$ of $A$ of finite measure,

(4) $\chi_{A} \chi_{C} \circ T^{2}=\chi_{C}$, i.e., $T_{A}^{2}=T_{A}$.

Conversely, suppose (i) and (ii) hold. Then (i) implies that $T$ maps $A$ into $A$ so that $A \subseteq T^{-1} A$. Combining this with (ii) we have

(5) $w w \circ T=h E(\bar{w}) \circ T^{-1} w \circ T=h E\left(\bar{w} w \circ T^{2}\right) \circ T^{-1}=h E\left(\left|w^{2}\right|\right) \circ T^{-1}$, since $\bar{w} w \circ T^{2}=\bar{w} \chi_{A} w \circ T^{2}=\bar{w} w \circ T_{A}^{2}=\bar{w} w=|w|^{2}$. In particular, $A=\operatorname{supp} h E\left(|w|^{2}\right) \circ T^{-1}$. 
But it is always true that $\operatorname{ker} W_{T}=L^{2}\left(X \backslash \operatorname{supp} h E\left(|w|^{2}\right) \circ T^{-1}\right)$, so $W_{T} f=0$ for all $f \in L^{2}(X \backslash A)$. On the other hand, $W_{T}^{*} f=h E(\bar{w} f) \circ T^{-1}=0$ if $f \in L^{2}(X \backslash A)$. Finally for each $f \in L^{2}(A)$ we have $W_{T} f=w f \circ T=h E(\bar{w}) \circ T^{-1} f \circ T=h E\left(\bar{w} f \circ T^{2}\right) \circ T^{-1}=$ $h E\left(\bar{w} f \circ T_{A}^{2}\right) \circ T^{-1}=h E(\bar{w} f) \circ T^{-1}=W_{T}^{*} f$, so that $W_{T}$ is Hermitian.

\section{REFERENCES}

1. J. J. Bastian, A decomposition of weighted translation operators, Trans. Amer. Math. Soc. 224 (1976), 217-230.

2. J. T. Campbell and J. E. Jamison, On some classes of weighted composition operators, Glasgow Math. J. 32 (1990), 87-94.

3. J. T. Campbell, M. Embryo-Wardrop, R. Fleming and N. Sivaramakrishnan, Normal and quasinormal weighted composition operators, pre-print.

Department of Mathematical Sciences

MEMPHIS STATE UNIVERSITY

Memphis, Tennessee 38152

U.S.A. 